\title{
Hydrolytic Stability of New Amino Acids Analogues of Memantine
}

\author{
Aleksandra Tencheva ${ }^{1}$, Radoslav Chayrov ${ }^{1}$, Petko Mandjukov ${ }^{1}{ }^{1}$, Dancho Danalev ${ }^{2}$ and \\ Ivanka Stankova $1, *$ (D) \\ 1 Chemistry Department, South-West University "Neofit Rilski”, 2700 Blagoevgrad, Bulgaria; \\ sany900@abv.bg (A.T.); elektrik@abv.bg (R.C.); pmandjukov@abv.bg (P.M.) \\ 2 Biotechnology Department, University of Chemical Technology and Metallurgy, 8 blvd. Kliment Ohridski, \\ 1756 Sofia, Bulgaria; dancho.danalev@gmail.com \\ * Correspondence: ivastankova@abv.bg
}

Received: 28 July 2020; Accepted: 2 September 2020; Published: 16 September 2020

\begin{abstract}
In the present work, the hydrolytic stability of new memantine analogues modified with amino acids, at different $\mathrm{pH}$ corresponding to the human biological liquids and organs, was evaluated. Memantine is an uncompetitive N-methyl-D-aspartate receptor antagonist with low-to moderate-affinity. In addition, it is the first representative of a novel class of Alzheimer's disease (AD) medications acting on the glutamatergic system by blocking N-methyl-D-aspartate receptors. Generally, prodrugs are compounds aiming to improve stability of active fragment and to facilitate transportation across the cell membranes or lipid barriers. The investigated series of prodrugs include modified memantine with the following amino acids: alanine, $\beta$-alanine, glycine, phenylalanine, and valine. Hydrolytic stability was determined at two different $\mathrm{pH}$ values 2.0 and 7.4 at $37^{\circ} \mathrm{C}$, similar to those in the human stomach and blood plasma. Specially developed UV-VIS spectrophotometric method for quantification of the concentrations of unchanged compounds was applied in the kinetic studies. Val-MEM is the most stable in neutral medium and at $37^{\circ} \mathrm{C}$ compound with $t_{1 / 2}=50.2 \mathrm{~h}$. The compound Phe-MEM has also very good hydrolytic stability with $t_{1 / 2}=29.6 \mathrm{~h}$. The order of other compounds is: Val-MEM $\gg$ Phe-MEM $\gg$ Ala-MEM $\approx$ Val-MEM $>\beta$-Ala-MEM. Ala-MEM and Gly-MEM are the most stable compounds at acid condition with almost identical values for $t_{1 / 2}=17.8 \mathrm{~h}$ and $t_{1 / 2}=16.3 \mathrm{~h}$, respectively. The stability of tested compounds in acid conditions are relatively less than in neutral one. They are ordered as follows: Ala-MEM $\approx$ Gly-MEM $>$ Val-MEM $\approx$ Phe-MEM $\approx \beta$-Ala-MEM. All compounds have relatively good hydrolytic stability of more than $10 \mathrm{~h}$ at both neutral and acid conditions, which is quite enough in order to pass in the blood circulation and to be used as a potential antimicrobial agent.
\end{abstract}

Keywords: amino acids; memantine; hydrolytic stability; prodrug; physiological $\mathrm{pH}$; non-linear calibration; first order kinetics

\section{Introduction}

The structure of adamantane influences its atypic physicochemical properties, good thermal and oxidative stability, extreme lipophilicity, and low energy [1,2]. Memantine (MEM) is an uncompetitive $\mathrm{N}$-methyl-D-aspartate receptor (NMDAR) antagonist with low- to moderate-affinity [3]. It was initially synthesized by Eli Lilly company and patented in 1968 as a derivative of amantadine [4]. In various studies, the memantine was reported to be a neuroprotective agent that positively impacts both neurodegenerative and vascular processes [5]. While excessive levels of glutamate result in neurotoxicity, in part through the over-activation of N-methyl-D-aspartate receptors, memantine-as a partial NMDARs antagonist, blocks these receptors to normalize the glutamatergic system and 
ameliorate cognitive and memory deficits [6]. Memantine contains three rings with a bridgehead amine $\left(-\mathrm{NH}_{2}\right)$ function, which under physiological conditions carries a positive charge $\left(-\mathrm{NH}_{3}{ }^{+}\right)$capable to bind on or near the $\mathrm{Mg}^{2+}$ site in the NMDARs-associated channel [7]. The NMDARs participates in regulation of synaptic plasticity and plays a role in learning and memory. Memantine is able to modulate the pathologic activation of NMDARs, presumably occurring in the process of AD, whilst retaining physiological activity important for learning and memory [8]. Memantine can replace $\mathrm{Mg}^{2+}$ during activation of NMDARs by interaction with NMDARs with a higher affinity than $\mathrm{Mg}^{2+}$ ions. It is also able to inhibit the prolonged influx of $\mathrm{Ca}^{2+}$ ions, which are responsible for excitotoxicity of neurons. The low affinity and rapid off-rate kinetics of memantine preserve the physiological function of the receptor, as it can still be activated by the relatively high concentration of glutamate release following depolarization of the presynaptic neuron $[9,10]$. Like other adamantane analogues, the most common side effects with memantine are dizziness, headache, constipation, drowsiness, and high blood pressure [11].

In modern medicine, the main problem for drug delivery is passing through cell membrane as well as transfer to the target cell. Prodrugs are bioreversible derivatives of drug molecules used to overcome some barriers to the utility of the parent drug molecule [12]. Classical prodrug design often represents a nonspecific chemical approach to mask undesired drug properties such as limited bioavailability, lack of site specificity, and hydrolytic as well as chemical instability [13]. One possible approach to facilitate crossing of biologically active compounds through the cell membrane is to bond them to specific transport molecules such as amino acids or peptides [14]. There are many examples in the literature for modification of known medical drugs with amino acids, which lead to increasing biological activity and often decreasing parent molecule toxicity [15]. Amino acids are involved in many cellular metabolic and signaling pathways, so the effects of altered amino acid metabolism in $\mathrm{AD}$ brain are far-reaching [16]. Taking into account all mentioned above, new memantine analogues with various amino acids-alanine, $\beta$-alanine, glycine, phenylalanine, and valine were synthesized as potential agents for treatment of illness related to cognitive and memory deficits.

There have been relations between Alzheimer's disease (AD) and infections that cause a long-term activation of the immune system, a process known as chronic inflammation. In our previous work, the investigated amino acid memantine analogs showed inhibitory effects against model Gram-positive (Staphylococcus aureus NBIMCC (6538) and Bacillus megaterium) and Gram-negative bacteria (Escherichia coli (NBIMCC 3397) and Salmonella enterica (NBIMCC 869)) as well as yeasts (Rhodotorula sp. (BF 16-25) and Candida lusitaniae (BF 74-4)) [17]. Comparing analysis of antimicrobial activity shows that compounds Gly-MEM and Val-MEM show inhibition activity against Gram (-) bacteria. Compound Val-MEM also inhibits proliferation of model strain Gram (+) bacteria Bacillus megaterium. Any of tested compounds do not show antifungal activity against used model strains yeasts.

Thus, the newly synthesized molecules have double effect and could be attractive as a possible alternative for treatment of diseases like $\mathrm{AD}$ and infections. The obtained analogues were tested in conditions corresponding to stomach $\mathrm{pH} 2.0$ and human plasma $\mathrm{pH} 7.4$ at $37^{\circ} \mathrm{C}$.

\section{Materials and Methods}

\subsection{Reagents and Methods}

The buffer components $\mathrm{HCl}, \mathrm{KCl}, \mathrm{NaCl}, \mathrm{Na}_{2} \mathrm{HPO}_{4}$ and $\mathrm{H}_{3} \mathrm{PO}_{4}$ were purchased from Sigma Aldrich (St. Louis, MO, USA). The kinetic study were performed on Agilent 8453 UV/Vis Spectrophotometer Agilent Technologies, 5301 Stevens Creek Blvd, Santa Clara, CA 95051, United States. Software: UV-Visible ChemStation. Light sources: low pressure deuterium lamp (wavelength range from $190 \mathrm{~nm}$ to approximately $800 \mathrm{~nm}$ ); low-noise tungsten lamp (wavelength range from $370 \mathrm{~nm}$ to $1100 \mathrm{~nm}$ ). Detector: Diode Array: 1024 individual photodiodes and control circuits etched onto a semiconductor chip. Cuvette: $10 \mathrm{~mm}$ cuvette 


\subsection{Solutions}

The buffer solutions used in determination of hydrolytic stability of aim compounds were prepared according to the European Pharmacopoeia, 6th Edition as follows:

(i) buffer $\mathrm{pH} 2.0-6.57 \mathrm{~g} \mathrm{KCl}$ were dissolved in water $\left(\mathrm{CO}_{2}\right.$ free $)$ and $119.0 \mathrm{~mL} 0.1 \mathrm{~mol} / \mathrm{L} \mathrm{HCl}$ were added. The obtained solution was diluted to $1000.0 \mathrm{~mL}$ with $\mathrm{dH}_{2} \mathrm{O}$;

(ii) buffer $\mathrm{pH} 7.4-2.38 \mathrm{~g} \mathrm{Na}_{2} \mathrm{HPO}_{4}, 0.19 \mathrm{~g} \mathrm{KH}_{2} \mathrm{PO}_{4}$ and $8.0 \mathrm{~g} \mathrm{NaCl}$ were dissolved in $\mathrm{dH}_{2} \mathrm{O}$. The obtained solution was diluted to $1000.0 \mathrm{~mL}$ with $\mathrm{dH}_{2} \mathrm{O}$;

(iii) standard solutions for UV-VIS-NIR spectrometry.

\subsection{Kinetic Study}

The kinetic study was performed by measuring the concentration of the memantine compounds and their decomposition products every $30 \mathrm{~min}$ for a period of $300 \mathrm{~min}$ under defined conditions. UV-VIS Spectrophotometric method was used for analyte quantification at relevant $\lambda_{(\max )}$ for the respective compound (range 205-220 nm).

For this purpose, memantine amides containing amino acids (Ala, $\beta$-Ala, Gly, Phe and Val) were incubated at $\mathrm{pH} 2.0$ and $\mathrm{pH}$ 7.4. The stock solutions of the respective compounds were prepared immediately before the stability studies. Aliquots $(9.8 \mathrm{~mL})$ of the buffer were placed in a screw-capped vial and tempered at $37^{\circ} \mathrm{C}$. A studied compound stock solution $(0.2 \mathrm{~mL})$ was added to the buffer. The vial was placed in a magnetic stirrer with a bath at the same temperature and agitated at $60 \mathrm{rpm}$ for $300 \mathrm{~min}$. Each sample was directly analyzed by UV-VIS-NIR spectrometry.

\subsection{General Methodology for Peptide Synthesis in Solution Using 2-(1H-Benzotriazole-1-yl)-1,1,3,3- Tetramethylaminium Tetrafluoroborate (TBTU) as a Coupling Reagent}

One eq of memantine was dissolved in in a minimal amount of dichloromethane. The corresponding amino acids Boc-AA-OH (1.3 eq), TBTU (1.3 eq) and TEA (1.3 eq), were added to the solution. The reaction mixture was stirred for $24 \mathrm{~h}$ at a room temperature. After $24 \mathrm{~h}$, the solvent was evaporated. The obtained product was extracted by $3 \times 25 \mathrm{~mL}$ dichloromethane. Further, combined organic layers were washed with $10 \%$ citric acid in water $(3 \times 25 \mathrm{~mL}), 5 \% \mathrm{NaHCO}_{3}(3 \times 25 \mathrm{~mL})$ and water $(3 \times 25 \mathrm{~mL})$. Organic layers were finally dried on anhydrous $\mathrm{Na}_{2} \mathrm{SO}_{4}$, and solvent was removed under vacuum.

\subsection{General Methodology for Boc-Group Deprotection}

One eq of corresponding Boc-AA-memantine was dissolved in 10-fold excess of trifuoroacetic acid (TFA) at $0{ }^{\circ} \mathrm{C}$. The reaction mixture was stirred until Boc group running out (the chromatographic control was carried out in systems chloroform-methanol 95:5 ratio). The solvent was evaporated and the residue was dissolved in $10 \mathrm{~mL}$ methanol. Twenty-five percent ammonia solution was added until the $\mathrm{pH}$ reached approximately 9. The solvent was evaporated under vacuum. Obtained crystals were dissolved in ethyl acetate and washed with water $(3 \times 25 \mathrm{~mL})$. The organic layer dried on anhydrous $\mathrm{Na}_{2} \mathrm{SO}_{4}$ and solvent was removed. The yield on each compound is various, but it is within $65-82 \%$.

\section{Results}

This investigation aimed to study hydrolytic stability of memantine analogues with amino acids at $\mathrm{pH} 2.0$ and $\mathrm{pH} 7.4$ at $37^{\circ} \mathrm{C}$. UV-VIS Spectrophotometric method was specially developed for quantification of the amide concentrations. The hydrolytic stability of memantine derivatives: alanyl-memantine (1), $\beta$-alanyl-memantine (2), glycyl-memantine (3), phenylalanyl-memantine (4), and valyl-memantine (5) was studied under experimental conditions of biological relevance, i.e., at $\mathrm{pH} 2.0$ and $\mathrm{pH} 7.4$, at a temperature of $37^{\circ} \mathrm{C}[15,16]$. All studied compounds (Figure 1) were synthesized using TBTU as a coupling reagent. 


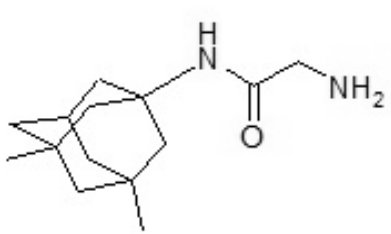

(1)

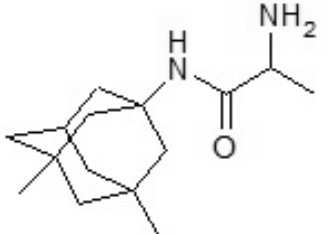

(2)

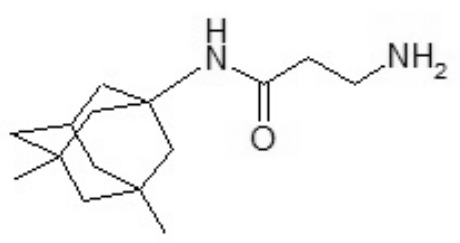

(3)

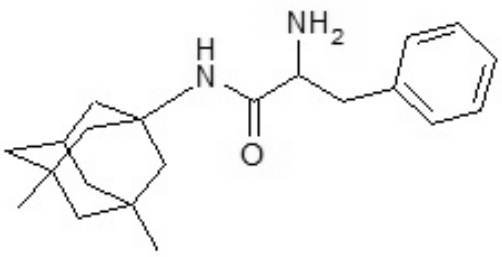

(4)

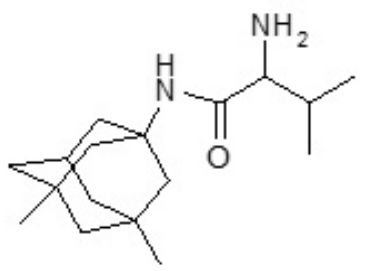

(5)

Figure 1. Memantine analogues with amino acids: alanyl-memantine Ala-MEM (1), $\beta$-alanylmemantine $\beta$ Ala-MEM (2), glycyl-memantine Gly-MEM (3), phenylalanyl-memantine Phe-MEM (4) and valyl-memantine Val-MEM (5).

In most of the cases, the calibration function in UV-VIS spectrometry is linear and rather stable. Exceptions are relatively rare, but existing. In the region of high concentrations, negative deviations from the Beer's law are always expected. Six to seven calibration standard solutions, covering the working range, were prepared for all substances studied in exactly the same way as for the kinetic study described above. All calibration points in the higher concentration region, exceeding $15 \%$ negative deviation from the straight line were rejected. The nature of the experiment, the possibility to prepare blank solution precisely matching the matrix in all standards and samples measured, and automatic blank correction used allows including the point at concentration 0 in calculation of the regression line. It is generally not needed if the calibration function is linear. However, in the case of nonlinearity, it is important and recommended for correct calculation of the regression coefficients.

Correct selection of calibration model and estimation of the concentration of the studied compounds is a problem of crucial importance for the reliability of the kinetic studies. Preliminary kinetic experiment was performed to ensure that all data points are situated within the working range of the corresponding calibration function. If some of the data points appear to be outside the concentration range between the lowest and the highest calibration standard, either calibration range or concentration of the solution used in kinetic study were respectively adjusted.

The linearity of the calibration functions was examined visually by the residual deviation plot graphical method [18]. It is recommended by IUPAC for quick evaluation, as a part of the calibration validation procedure. This is due to the fact that frequently used statistical tests are generally not sensitive to the distribution of the deviations along the regression line. When the statistic, related to some of the other statistical tests, is close to the critical value, the residual deviation plot is helpful for making the final decision. Additionally, it is a simple qualitative homoscedasticity test. When the lowest and highest points of the calibration graphic are on the same side of the most probable straight line, while the middle part is on the other side, this is a clear indication that the real function is non-linear. In such cases, second order polynomials were tested as a calibration model. The final decision for use of linear or non-linear calibration functions was based on the Fisher's F-test [19]. In most of the cases, the calibration function appeared to be non-linear with only one exception-the case of Ala-MEM, where the function was suggested as linear. Since the calibration model based on second order polynomial function could be successfully validated, more complicated mathematical functions were not tested. The finally selected calibration equations for all compounds studied are listed in Table 1. All kinetic experiments were performed under repeatability conditions, immediately after the respective calibration, in order to avoid the effect of any potential variability between days. 
Table 1. Regression coefficients squared correlation coefficients, and concentration range for the calibration functions of the type: $y=a_{2} x^{2}+a_{1} x+a_{0}$.

\begin{tabular}{|c|c|c|c|c|c|c|}
\hline \multirow{2}{*}{ №. } & \multirow{2}{*}{ Compound } & \multicolumn{3}{|c|}{ Calibration Equations } & \multirow{2}{*}{$\mathbf{R}^{2}$} & \multirow{2}{*}{ Concentration Range $[\mathrm{g} / \mathrm{L}]$} \\
\hline & & $\mathbf{a}_{2} *$ & $\mathbf{a}_{1}$ & $\mathbf{a}_{0}$ & & \\
\hline (1) & Ala-MEM & 0 & 6.7698 & 0.0243 & 0.9976 & $0.01-0.10$ \\
\hline (2) & ßAla-MEM & -18.855 & 9.878 & 0.0253 & 0.9976 & $0.01-0.08$ \\
\hline (3) & Gly-MEM & -45.433 & 17.52 & 0.0351 & 0.9929 & $0.01-0.08$ \\
\hline (4) & Phe-MEM & -869.25 & 78.599 & 0.0039 & 0.9999 & $0.01-0.12$ \\
\hline (5) & Val-MEM & -69.123 & 14.123 & 0.0144 & 0.9974 & $0.01-0.12$ \\
\hline
\end{tabular}

The residual deviation plots for representative cases of Val-MEM for non-linear and Ala-MEM for linear are shown in Figure 2.
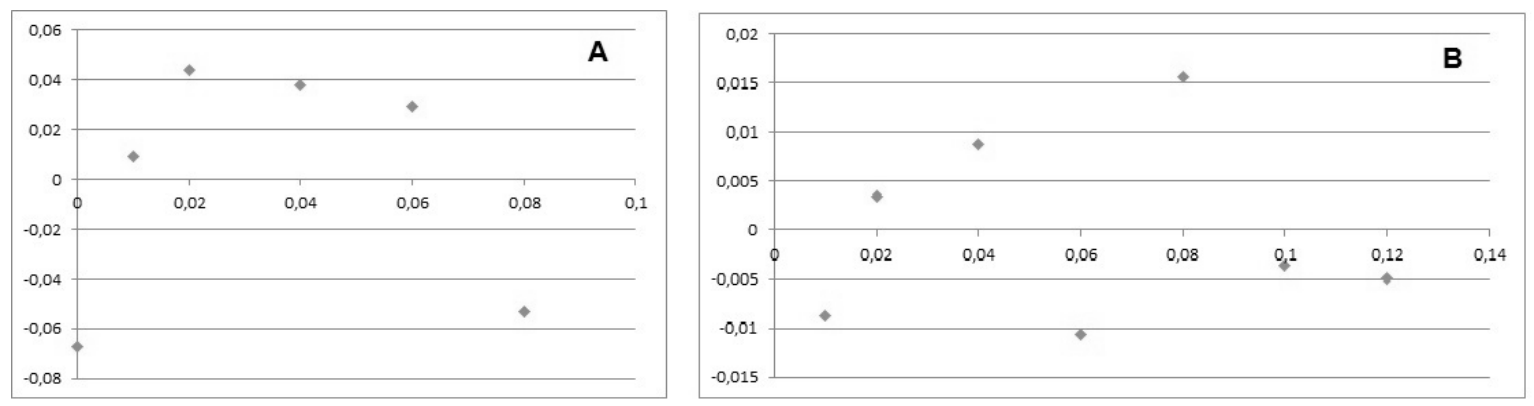

Figure 2. Residual deviation plot: (A) for Val-MEM as a nonlinear example; (B) for Ala-MEM.

Respective comparisons between linear and second order polynomial calibration graphics for the same examples are presented in Figure 3.
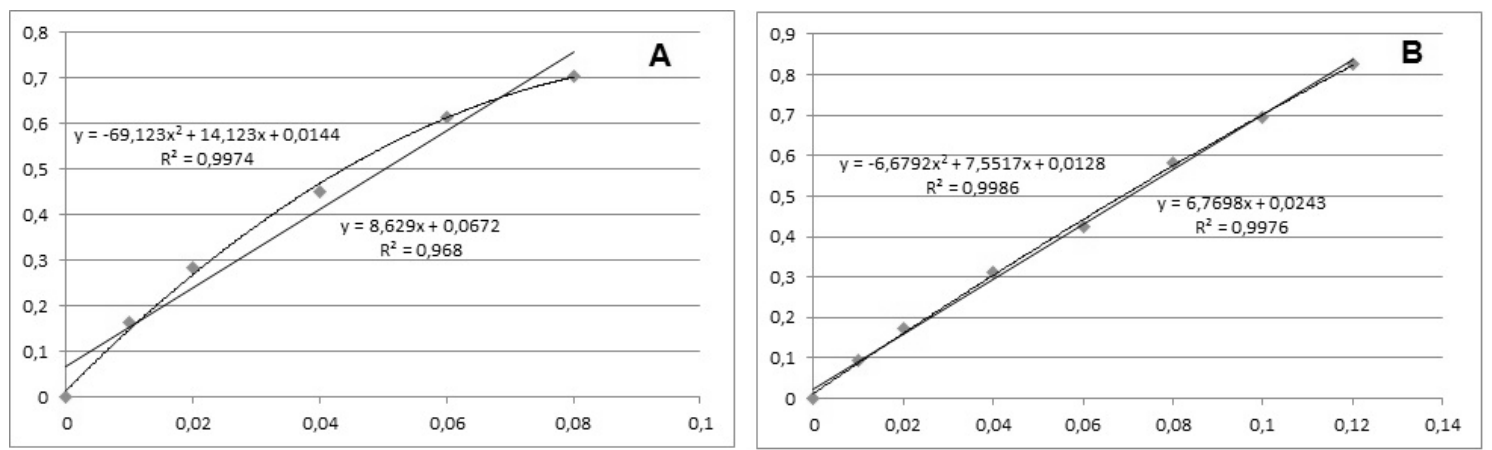

Figure 3. Calibration graphics: (A) Non-linear calibration graphics for Val-MEM; (B) Linear calibration graphics for Ala-MEM.

\section{Discussion}

Correct selection of calibration model and estimation of the concentration of the studied compounds is a problem of crucial importance for the reliability of the kinetic studies. All calibration standards were prepared using the same compounds as in kinetic as described above. Preliminary kinetic experiment was performed to ensure that all data points are situated within the working range of the corresponding calibration function. If some of the data points appear to be outside the concentration range between the lowest and the highest calibration standard, either calibration range or concentration of the solution used in kinetic study were respectively adjusted.

The linearity of the calibration functions was examined visually by the residual deviation plot graphical method [18]. When the lowest and highest points of the calibration graphic are on the 
same side of the most probable straight line, while the middle part is on the other side, this is a clear indication that the real function is non-linear. In such cases, second order polynomials were tested as a calibration model. The final decision for use of non-linear calibration functions was confirmed by the Fisher's F-test [19]. In most of the cases, the calibration function appeared to be non-linear with only one exception - the case of Ala-MEM where the function suggested as linear. According to the literature data and previous studies, in most of the cases, the hydrolysis of drugs is following zero or first order kinetics. Zero order implies concentration-independent rate and linear function: $C$ vs. $t$. In case of first or pseudo-first order kinetics, the reaction rate depends on the concentration of one reactant and linearity can be achieved in coordinates: $\ln \left(C / C_{0}\right)$ vs. $t$. The linearity of the kinetic curves in the present study was evaluated by Pearson's correlation coefficient and Student's t-test. It should be noted that practically always when $R^{2} \geq 0.99$, the application of other linearity tests also provides positive results. These tests were applied both for examination of zero order (first function) first or pseudo first order (second function). In both cases, the slope of the regression line is equal to the rate constant of the process.

It should be noted that the conclusion based on formal kinetic evaluation of the reaction order might be significantly complicated if the concentration of the studied compound varies in a narrow concentration range. In such a case, the function $\ln (C)$ trends to linear. Thus, both regression functions might show similar linearity estimated by values of $R^{2}$ and the statistic for Students t-test. To distinguish between zeroth and first order kinetics, additional information is needed.

One of the possible solutions is to compare kinetic curves $C$ vs. $t$ build for different initial concentrations of the studied compounds. If the process is of zeroth order, the slope of the curve should be independent from $C_{0}$ value. To the contrary, in the case of first order reaction, the slope of the curve in the region close to $t=0$ should be different for different initial concentrations.

Both models were tested for all compounds at $\mathrm{pH}=1$ and in buffer solution $(\mathrm{pH}=7)$.

In the present study, the $R^{2}$ values for linear and logarithmic kinetic curves were not significantly different, as could be expected considering the narrow concentration range. Comparing data from the preliminary experiments and the final investigations, differences were found between the slopes of the linear kinetic curves. Since the rate constant in all kinetic models should be independent from the concentration changes, first or pseudo-first order kinetics was assumed.

Values of the rate constants were obtained as a slope of the linear regression line in coordinates $\ln \left(C / C_{0}\right)$ vs. $t$. Standard deviation of the slope $\left(s_{a_{1}}\right)$ was calculated according to Equation (1) [20]:

$$
s_{a_{1}}=s_{R} \sqrt{\frac{1}{\sum_{i=1}^{n} x_{i}^{2}-\frac{1}{n}\left(\sum_{i=1}^{n} x_{i}\right)^{2}}}
$$

where: $x_{i}$ is the time value for the $i$ th data point from each kinetic curve, and $S_{R}$ is the regression standard deviation, calculated using Equation (2).

$$
s_{R}=\sqrt{\frac{\sum_{i=1}^{n}\left(y_{i}-\hat{y}_{i}\right)^{2}}{n-c}}
$$

where: $y_{i}$ and $\hat{y}_{i}$ are respectively the measured and evaluated, according to regression equation, absorbance values for the $i$ th data point from the each kinetic curve, $n$ is the number of points and $c$ is the number of coefficients in the regression equation (in the present case: $c=2$ ).

Since the measured value is the slope of the linear regression line, its standard deviation may be assumed as an estimate of the combined standard measurement uncertainty [21] of the rate constant $\left(u_{c}\left(k_{r}\right)\right)$.

Reaction half-time $\left(t_{1 / 2}\right)$ can be estimated according to Equation (3):

$$
t_{1 / 2}=\ln \frac{2}{k_{r}}
$$


where $k_{r}$ is the rate constant of the reaction. Applying the uncertainty propagation law [21] to Equation (3), an expression can be derived for evaluation of the combined standard measurement uncertainty of the reaction half-time $\left(u_{c}\left(t_{1 / 2}\right)\right)$.

$$
u_{c}\left(t_{1 / 2}\right)=\ln \frac{k_{r}}{2} \times\left(\frac{u\left(k_{r}\right)}{k_{r}}\right)
$$

Expanded uncertainty (half-width of the confidence interval) $[20,21]$ for the rate constant $\left(k_{r}\right)$ and the reaction half-time $\left(t_{1 / 2}\right)$ can be calculated according to Equation (5):

$$
U=k \times u_{c}
$$

where $k$ is coverage factor and $u_{c}$ is the corresponding combined standard measurement uncertainty. Typically, for confidence probability 0.95 (level of significance 0.05 ): $k=2$.

The results from the kinetic investigations are summarized in Table 2.

Table 2. Rate constants for degradation processes of memantine analogues, reaction half-times and correlation coefficient of the regression line in coordinates $\ln \left(C / C_{0}\right)$ vs. $t$. All experiments were carried out at $37^{\circ} \mathrm{C}$.

\begin{tabular}{ccccc}
\hline No & Compound/Media & Rate Constant $\boldsymbol{k}_{\boldsymbol{r}}$ & $\begin{array}{c}\text { Reaction Half-Time } \\
\boldsymbol{t}_{\mathbf{1} / \mathbf{2}} \mathbf{~}\end{array}$ & $\begin{array}{c}\text { Correlation Coefficient } \\
\mathbf{R}^{\mathbf{2}}\end{array}$ \\
\hline$(1)$ & Ala-Mem/pH 2.0 & $-0.00103 \pm 0.00006$ & $17.8 \pm 0.5$ & 0.9919 \\
$(2)$ & BAla-Mem/pH 2.0 & $-0.00109 \pm 0.00004$ & $9.9 \pm 0.2$ & 0.9951 \\
$(3)$ & Gly-Mem/pH 2.0 & $-0.00065 \pm 0.00004$ & $16.3 \pm 0.7$ & 0.9908 \\
$(4)$ & Phe-Mem/pH 2.0 & $-0.00071 \pm 0.00006$ & $10.6 \pm 0.2$ & 0.9836 \\
$(5)$ & Val-Mem/pH 2.0 & $-0.00117 \pm 0.00006$ & $11.2 \pm 0.3$ & 0.9943 \\
$(1)$ & Ala-Mem/pH 7.4 & $-0.00023 \pm 0.00002$ & $12.7 \pm 0.4$ & 0.9928 \\
$(2)$ & BAla-Mem/pH 7.4 & $-0.00039 \pm 0.00002$ & $11.8 \pm 0.4$ & 0.9922 \\
$(3)$ & Gly-Mem/pH 7.4 & $-0.00091 \pm 0.00006$ & $9.9 \pm 0.6$ & 0.9908 \\
$(4)$ & Phe-Mem/pH 7.4 & $-0.00117 \pm 0.00014$ & $29.6 \pm 0.8$ & 0.9693 \\
$(5)$ & Val-Mem/pH 7.4 & $-0.00098 \pm 0.00006$ & $50.2 \pm 2.2$ & 0.9914 \\
\hline
\end{tabular}

Note: Half width of all confidence intervals are calculated as expanded uncertainty with coverage factor corresponding to 0.95 confidence probability $(k=2)$.

It was established that under the described experimental conditions, all amides underwent decomposition by hydrolysis. The hydrolysis followed apparent first order kinetics, and the rate constants $(\mathrm{k})$ were obtained as slopes from the semi-logarithmic plots of the unchanged ester concentration versus time. The chemical stability was assessed by means of the decomposition half-times of live $t_{1 / 2}=\ln 2 / \mathrm{k}$ (Table 2 ).

Hydrolytic stability of measurements revealed that the compounds were relatively stable at acid pH (Figure 4). 


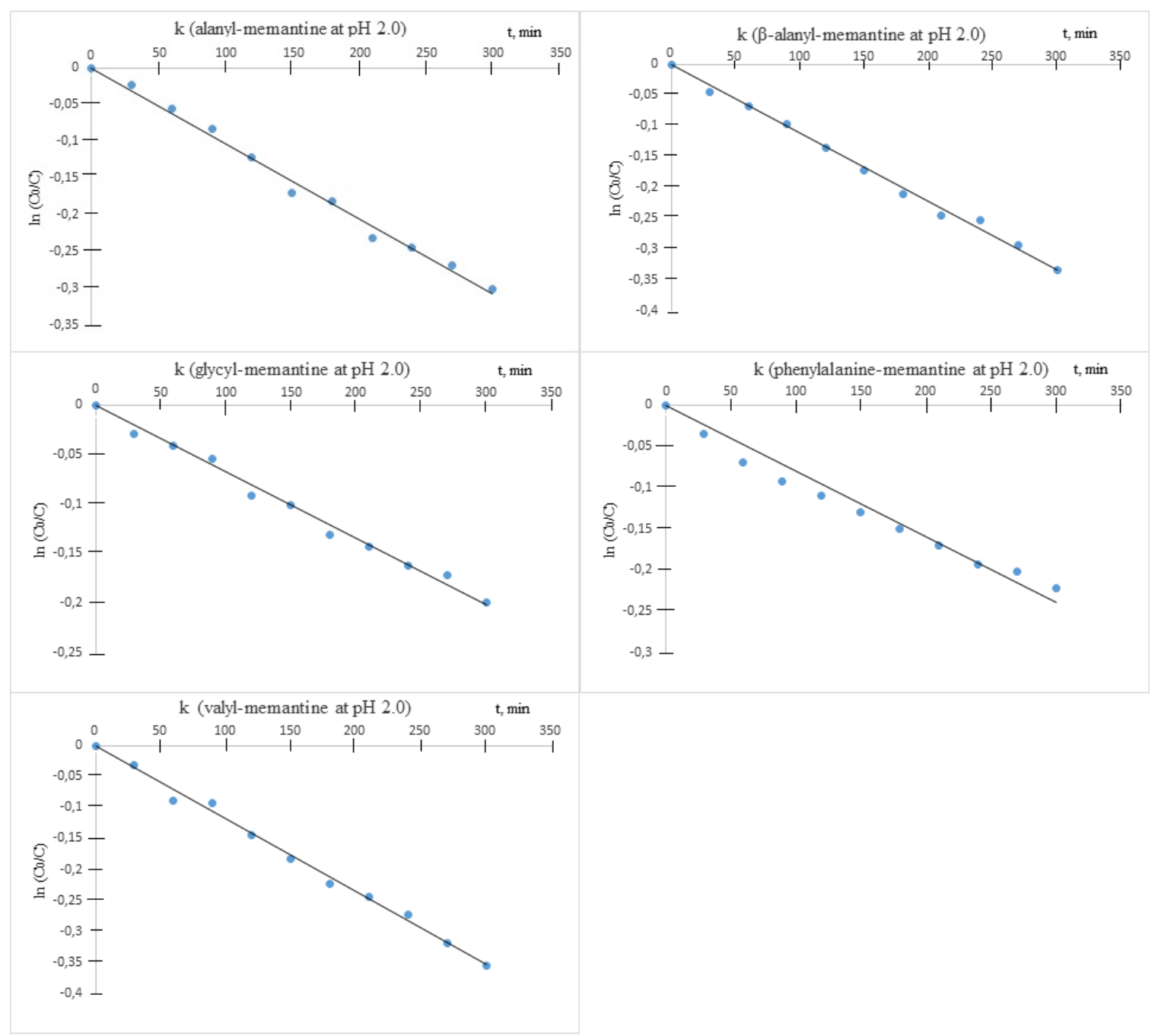

Figure 4. Changing of concentration of the examined memantine derivatives with time at $\mathrm{pH}$ 2.0.

Under these conditions, all of the observed half-lives were more than $10 \mathrm{~h}$. All tested compounds were also stable at $\mathrm{pH} 7.4$ (Figure 5). 


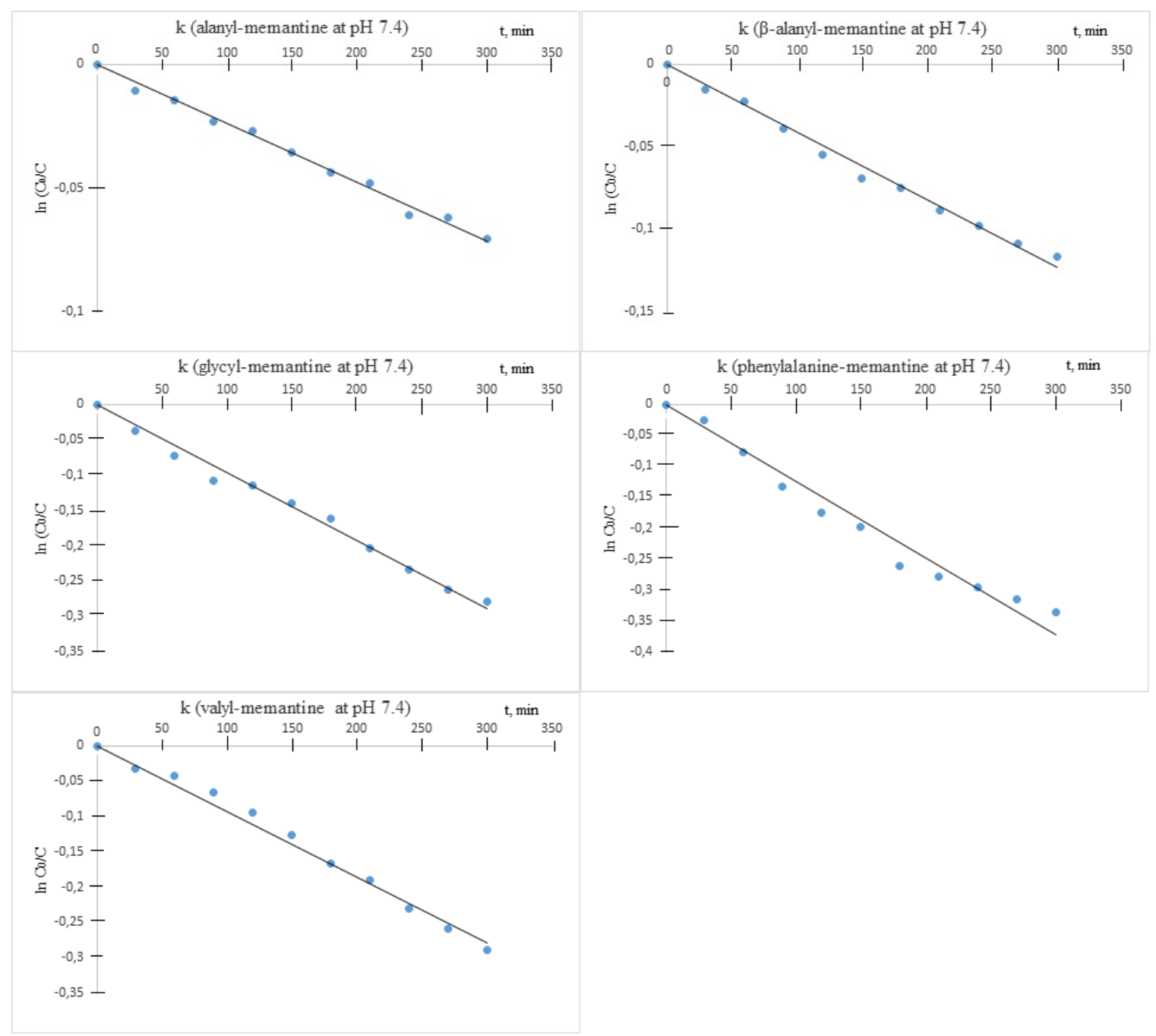

Figure 5. Changing of concentration of the examined memantine derivatives with time at pH 7.4.

\section{Conclusions}

The hydrolytic stability of hybrid molecules between memantine and several amino acids under experimental conditions simulating some relevant biological media ( $\mathrm{pH} 2.0$ and $\mathrm{pH} 7.4$, $37^{\circ} \mathrm{C}$ ) was studied using the developed UV-VIS spectrophotometric method. Val-MEM is the most stable in neutral medium and at $37{ }^{\circ} \mathrm{C}$ compound with $t_{1 / 2}=50.2 \mathrm{~h}$. The compound Phe-MEM has also very good hydrolytic stability with $t_{1 / 2}=29.6 \mathrm{~h}$. The order of other compounds is: Val-MEM $\gg$ Phe-MEM $\gg$ Ala-MEM $\approx$ Val-MEM $>\beta$-Ala-MEM. The compound Val-MEM is the most stable in acid condition and the same one is the most active against model strains Gram (+) microorganism. Ala-MEM and Gly-MEM are the most stable compounds at acid condition with almost identical values for $t_{1 / 2}=17.8 \mathrm{~h}$ and $t_{1 / 2}=16.3 \mathrm{~h}$, respectively. The stability of tested compounds in acid conditions is relatively less than in the neutral one. They are ordered as follows: Ala-MEM $\approx$ Gly-MEM $>$ Val-MEM $\approx$ Phe-MEM $\approx \beta$-Ala-MEM. All compounds have relatively good hydrolytic stability of more than $10 \mathrm{~h}$ at both neutral and acid conditions, which is quite enough in order to pass in the blood circulation and to be used as an antimicrobial agent. In addition, our tests show that memantine derivatives containing aromatic amino acids possess an equivalent neuroprotective effect in comparison with these of the aliphatic amino acids.

Thus, some found correlations between biological activities and hydrolytic stability, which suggests that these compounds could be an attractive alternative for treatment of illness related to cognitive and memory deficits as well as bacterial infections. 
Author Contributions: Conception I.S., D.D. and P.M.; realization of experiments for hydrolytic stability, A.T. and R.C.; validation of developed method calculation of $t_{1 / 2}$, kinetic model and data interpretation R.C. and P.M.; methodology, I.S., writing — original draft preparation D.D., P.M., R.C. and I.S., writing—review and editing, D.D., P.M., R.C. All authors have read and agreed to the published version of the manuscript.

Funding: This work was supported by the Bulgarian National Science Fund (Project M/23-8).

Conflicts of Interest: The authors declare no conflict of interest.

\section{References}

1. Bauer, L.; Khullar, K.K. Carbon-sulfur cleavage of 1-adamantyl sulfides. J. Org. Chem. 1971, 36, 3038-3040. [CrossRef]

2. Halimehjani, A.Z.; Marjani, K.; Ashouri, A.; Amani, V. Synthesis and characterization of transition metal dithiocarbamate derivatives of 1-aminoadamantane: Crystal structure of (N-adamantyldithiocarbamato) nickel (II). Inorg. Chim. Acta 2011, 373, 282-285. [CrossRef]

3. Tariot, P.N.; Farlow, M.R.; Grossberg, G.T.; Graham, S.M.; McDonald, S.; Gergel, I.; Memantine study group. Memantine treatment in patients with moderate to severe Alzheimer disease already receiving donepezil: A randomized controlled trial. JAMA 2004, 291, 317-324. [CrossRef] [PubMed]

4. Di Iorio, G.; Baroni, G.; Lorusso, M.; Montemitro, C.; Spano, M.C.; Di Giannantonio, M. Efficacy of memantine in schizophrenic patients: A systematic review. J. Amino Acids 2017. [CrossRef] [PubMed]

5. $\quad$ Olivares, D.; Deshpande, V.K.; Shi, Y.; Lahiri, D.K.; Greig, N.H.; Rogers, J.T.; Huang, X. N-methyl D-aspartate (NMDA) receptor antagonists and memantine treatment for Alzheimer's disease, vascular dementia and Parkinson's disease. Curr. Alzheimer Res. 2012, 9, 746-758. [CrossRef] [PubMed]

6. Ghosh, A. Learning more about NMDA receptor regulation. Science 2002, 295, 449-451. [CrossRef]

7. Peskind, E.R.; Potkin, S.G.; Pomara, N.; Ott, B.R.; Graham, S.M.; Olin, J.T. Memantine MEM-MD-10 study group. memantine treatment in mild to moderate alzheimer disease: A 24-week randomized, controlled trial. Am. J. Geriatr. Psychiatry 2006, 14, 704-715. [CrossRef]

8. Wu, T.Y.; Chen, C.P. Dual action of memantine in Alzheimer disease: A hypothesis. Taiwan. J. Obstet. Gynecol. 2009, 48, 273-277. [CrossRef]

9. Parsons, C.G.; Stöffler, A.; Danysz, W. Memantine: A NMDA receptor antagonist that improves memory by restoration of homeostasis in the glutamatergic system-too little activation is bad, too much is even worse. Neuropharmacology 2007, 53, 699-723. [CrossRef] [PubMed]

10. Stella, V.J. Prodrugs as therapeutics. Expert Opin. Ther. Pat. 2004, 14, 277-280. [CrossRef]

11. Govorkova, E.A.; Hong-Bin, F.; Ming, T.; Webster, G.R. Neuraminidase inhibitor-rimantadine combinations exert additive and synergistic anti-influenza virus effects in MDCK cells. Antimicrob. Agents Chemother. 2004, 48, 4855-4863. [CrossRef] [PubMed]

12. Han, H.-K.; Amidon, G.L. Targeted prodrug design to optimize drug delivery. AAPS Pharm. Sci. 2000, 2, 48-58. [CrossRef]

13. Beauchamp, L.M.; Orr, G.F.; de Miranda, P.; Burnette, T.; Krenitsky, T.A. Amino acid ester prodrugs of aciclovir. Antivir. Chem. Chemother. 1992, 3, 157-164. [CrossRef]

14. Shibnev, V.A.; Garaev, T.M.; Finogenova, M.P.; Shevchenko, E.S.; Burtseva, E.I. Some pathways to overcoming drug resistance of influenza a virus to adamantane derivatives. Pharm. Chem. J. 2012, 46, 1-5. [CrossRef]

15. Griffin, J.W.; Bradshaw, P.C. Amino acid catabolism in Alzheimer's disease brain: Friend or foe? Oxid. Med. Cell. Longev. 2017. [CrossRef]

16. Zacchigna, M.; Di Luca, G.; Maurich, G.V.; Boccu, E. Syntheses, chemical and enzymatic stability of new poly(ethyleneglycol)-acyclovir prodrugs. Farmaco 2002, 57, 207-214. [CrossRef]

17. Chayrov, R.; Tencheva, A.; Sbirkova-Dimitrova, H.; Shivachev, B.; Kujumdzieva, A.; Nedeva, T.; Stankova, I. Synthesis, antibacterial, and antifungal activities of hybrid molecules based on alzheimer disease drugs and bearing an amino acid fragment. Proceedings 2019, 41, 23. [CrossRef]

18. Danzer, K.; Currie, L. A Guidelines for calibration in analytical chemistry. Part, I. Fundamentals and single component calibration (IUPAC Recommendations 1998). Pure Appl. Chem. 1998, 70, 993-1014. [CrossRef]

19. Miller, J.N.; Miller, J.C. Statistics and Chemometrics for Analytical Chemistry, 6th ed.; Pearson Education Ltd.: Harlow, UK, 2010. 
20. Joint Committee for Guides in Metrology. International Vocabulary of Metrology-Basic and General Concepts and Associated Terms (VIM3) JCGM 200:2012. Available online: https://www.bipm.org/utils/ common/documents/jcgm/JCGM_200_2012.pdf (accessed on 9 June 2020).

21. Joint Committee for Guides in Metrology. Evaluation of Measurement Data-Guide to the Expression of Uncertainty in Measurement (GUM) JCGM 100:2008. Available online: https://www.bipm.org/utils/common/ documents/jcgm/JCGM_100_2008_E.pdf (accessed on 9 June 2020).

(C) 2020 by the authors. Licensee MDPI, Basel, Switzerland. This article is an open access article distributed under the terms and conditions of the Creative Commons Attribution (CC BY) license (http://creativecommons.org/licenses/by/4.0/). 